\title{
The effectiveness of vibration damper attached to the cable due to wind action
}

\author{
Irena Gołębiowska ${ }^{1}$, Maciej Dutkiewicz,"* \\ ${ }^{1}$ Department of Building Construction, University of Science and Technology in Bydgoszcz, ul. Prof. S. Kaliskiego 7, 85-796 \\ Bydgoszcz, Poland.
}

\begin{abstract}
In the paper the analysis of vibration of cable with attached damper is performed. Source of vibrations comes from the wind. The level of reduction of vibrations is also analysed. Damper attached to the cable is characterized by mass, stiffness and damping ratio. The paper also presents the equations of motion for the cable with damper, taking into account the initial parameters: cable cross-section and the location of the damper. The analysis is performed in time domain. It is discussed that in real situations the different types of cable vibration due to their amplitudes and frequencies is met: short-term oscillations of high amplitude, caused by a single impulse force, called bouncing cable, low amplitude and low wave length and the high frequency, called aeolian vibration, large oscillation amplitude, long wave length and low frequency, called galloping or dancing cables.
\end{abstract}

\section{Introduction}

\subsection{Bridge cable vibrations}

Due to construction benefits and architectural view in recent years a significant number of suspension and cable-stayed bridges were built. From the point of load and work of bridge, the wind load can be reason of vortex induced vibrations that can adversely effect on strength, durability of cables and connection failures $[1,2]$. In some cases local fatigue cracks are formed and further destruction of the structure is performed. Methods of reduction vibrations are applied to avoid such situations as cables have a very low level of damping an low bending stiffness [3]. Connection of cables to each other is another possible solution but this solution is used very rarely because of the adverse visual impact. Application of passive vibration damper, usually mounted at a distance not exceeding $5 \%$ of the length from the anchorage cable construction platform is another solution that is often used. Aerodynamic cable surface modification is another method of vibration reduction [1].

The selection of appropriate parameters of the vibration damper is associated with the characteristics of the damped structure working under the dynamic load. Cables perform uninterrupted vibrations both at minimum parameters of the damping system as well as when damping parameter is large, so it is essential to perform the tuning of the damping system [4].

\subsection{Transmission lines vibrations}

Vibrations of transmission lines due to wind actions are characterized by high frequency and small amplitudes of vibrations. They are called aeolian vibrations. Apart from them, oscillations of high amplitude and low frequency called galloping vibrations and secondary induced vibrations of average amplitude but lower than aeolian frequency are also observed. Aeolian vibrations are in the range of 3 to $50 \mathrm{~Hz}$ at wind speed from 0,5 to 7 $\mathrm{m} / \mathrm{s}$ acting perpendicular to the cable, which usually happens in open plots, in the area without buildings and infrastructure. The amplitude of oscillation does not exceed $2.5 \mathrm{~cm}[5,6]$. These vibrations are difficult to observe due to the fact that transission lines diameters are from 6 to $50 \mathrm{~mm}$, which exceeds the amplitudes of vibrations.

Galloping of cables appears at winds speed from 7 to 25 $\mathrm{m} / \mathrm{s}$. Cables oscillate according to first or second form as one or two half-waves. The frequency of oscillation is small, less than $1 \mathrm{~Hz}$ [5].

The maximum amplitude of vibrations occurs at resonance, and increases when the natural frequency is close to the forced frequency called Strouhal frequency. Vibrations can be controlled by the use of different dampers. The use of Stockbridge'a damper is a very popular solution.

Stockbridge damper is constructed from a flexible cable or rod and the masses at its ends [2]. The vibrations of the cable are passed through the clamp mounted the damper's rod to the cable and consequently the masses on the ends of the damper begin to vibrate. By careful selection of parameters of the damper such as mass at the ends of the rod and stiffness of the damper's rod, one can tune the optimal damping of the transmission energy line. First Stockbridge damper held at the ends of the rod masses made of concrete blocks. Nowadays steel masses at end of the damper's rod are used and galvanized steel is applied for damper's rod. In the modelling of Stockbridge damper the massless rod element is

* Corresponding author: macdut@utp.edu.pl 
analysed with masses at the ends. From the point of reduction of vibrations, the location of damper on the cable is significant [5].

The aeolian vibrations were analysed in the paper [8]. Analysis confirms that use of damper do not cause a dissipation of energy and reduction of vibrations in any case.

In the paper [6] the aplication of Stockbridge damper caused the feedback of the damper with the cable and disturbance of aeolian vibrations. Deterministic or probabilistic models are often applied among computational models. In the paper [5] a probabilistic model of the aeolian vibration with regard to fatigue strength was performed.

The purpose of the dampers is reduction of vibration by absorbing energy from the wind and stabilization of the movement of the cable to avoid deformation that can cause the damage. According to analysis performed in the paper [5] the value of safe limit deformation of the cable is $200 \mathrm{microns} / \mathrm{m}$. The effectiveness of the damper depends on its response to the wind force at a certain frequency.

\section{Modelling and analysis of cable's vibrations}

\subsection{Assumptions of the analysed system}

The issue of inextensible cable's free vibration was analyzed by Hajduk [7]. Vibration equations shown below fulfill the law of change of the amount of motion, law of conservation of mass and the law of change of the body deformation under load.

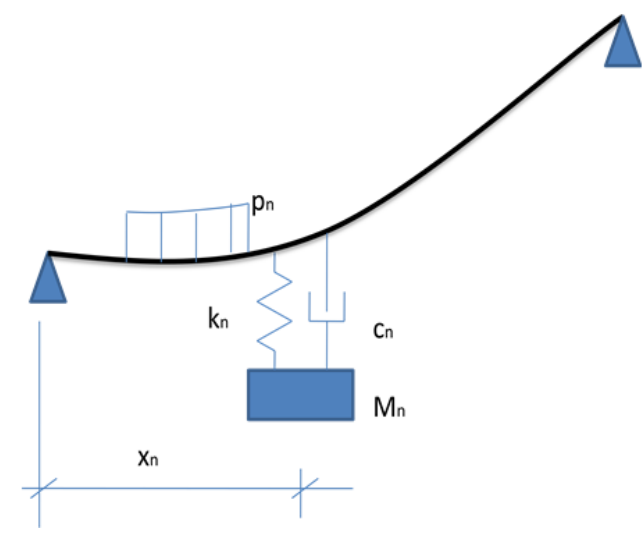

Fig.1. System of the cable with the damper

The general idea of the system equipped with the damper, that is the subject of the paper, is shown on figure 1. It is shown the cable of length 'l' under the load $\mathrm{p}_{\mathrm{n}}$ with the system of dampers represented by 'n-th' damper with parameters: stiffness $\mathrm{k}_{\mathrm{n}}$, damping $\mathrm{c}_{\mathrm{n}}$, mass $\mathrm{M}_{\mathrm{n}}, \mathrm{m}$ - mass of the cable distributed uniformly on the cable.

The purpose of the paper is to demonstrate the need of protection against vibrations of cables acting as bearing structures and proper selection of vibration dampers' parameters.
Numerical simulation is carried out for 20 seconds of vibration excitation, for span of the cable of $20 \mathrm{~m}$. The cable is modelled as a strand, non-displaceably fixed to the structure. It is assumed that path of the cable is inplane and the chord is horizontal. It is analysed the continous distribution of the mass along the length of the cable.

The analysis is limited to vertical vibrations occurring in the plane of the cable sag. According to Kirchhoff's hypothesis, because the sag of the cable is small, the effects of horizontal displacements is omitted. It is assumed that the material of the cable is linear-elastic and fulfill Hooke's law, i.e. the relationship between displacements and deformations is linear.

Taking into consideration the above assumptions the solution of dynamic displacement is taken in the form:

$$
w(x, t)=\sum_{j=1}^{k} \Phi(t) X_{j}(x)
$$

where:

$\Phi$ (t) is unknown function of time, treated as the coefficient of series:

$$
\Phi(t)=\phi_{0} e^{i \omega t}
$$

and $\phi_{0}$ is the amplitude coefficient of the function $\Phi(t)$, ' $i$ ' is the imaginary part of the complex number, $\omega$ is the forced frequency, $\mathrm{t}$ - means time of the analysed process. $X_{j}(x)$ shown in the equation no. 1 is function that meets all boundary conditions of the analysed problem:

$$
X_{j}(x)=\sum_{j=1}^{k} \sin \frac{j \pi x}{l}
$$

This assumption is made according to Galerkin's method for cable's vibration of an infinite number of degrees of freedom [7]. It is assumed that functions $X_{j}(x), \mathrm{j}=$ $1,2, \ldots, \mathrm{n}$, are linearly independent.

Vibration of the single damper is presented in the following equation:

$$
w_{1}(t)=\phi_{1} e^{i \omega t}
$$

where $\phi_{1}$ is the amplitude coefficient of the function $w_{1}(t)$.

\subsection{Solution and discussion}

Taking into account the approximation (1) and equations from (2) to (4), the equation of motion of the system can be written in Langrange'a coordinates:

$$
M \ddot{\Phi}(\mathrm{t})+\mathrm{C} \dot{\Phi}(\mathrm{t})+\mathrm{K} \Phi(\mathrm{t})=\mathrm{P}(\mathrm{t})
$$

where $\mathrm{M}, \mathrm{C}, \mathrm{K}$ are respectively matrices: mass, damping and stiffness, $\mathrm{P}(\mathrm{t})$ is the external load, i.e:

$M=\left[\begin{array}{ccc}m_{\text {red }} & \cdots & 0 \\ \vdots & \ddots & \vdots \\ 0 & \cdots & M_{n}\end{array}\right], C=\left[\begin{array}{ccc}C_{11} & \cdots & C_{1 n} \\ \vdots & \ddots & \vdots \\ C_{n 1} & \cdots & C_{n n}\end{array}\right]$, 


$$
K=\left[\begin{array}{ccc}
K_{11} & \cdots & K_{1 n} \\
\vdots & \ddots & \vdots \\
K_{n 1} & \cdots & K_{n n}
\end{array}\right], P=\left[\begin{array}{c}
p \\
\cdots \\
0
\end{array}\right]
$$

Orthogonality condition for the equation describing the vibrations of the cable takes the following form:

where:

$$
\int_{0}^{l} \sum_{p=1}^{s} \mathrm{~L}(w) X_{p}(x) d x=0
$$

$$
\begin{aligned}
L(w)=m \frac{\partial^{2} w(x, t)}{\partial t^{2}}-H_{s t} \frac{\partial^{2} w(x, t)}{\partial x^{2}} & \\
& -\frac{E F}{l} y^{\prime \prime}(x) \int_{0}^{l} \frac{\partial w(x, t)}{\partial x} y^{\prime}(x) d x \\
& +\gamma_{1} D \frac{\partial w(x, t)}{\partial t} \\
& -\frac{\eta F}{l} y^{\prime \prime}(x) \int_{0}^{l} \frac{\partial^{2} w(x, t)}{\partial x \partial t} y^{\prime}(x) d x \\
& +\sum_{n=1}^{m} k_{n}\left(w(x, t)-w_{n}(t)\right) \delta\left(x-x_{n}\right) \\
& +\sum_{n=1}^{m} c_{n}\left(\frac{\partial w(x, t)}{\partial t}-\frac{\partial w_{n}(t)}{\partial t}\right) \delta(x \\
& \left.-x_{n}\right)+p(x, t)(8)
\end{aligned}
$$

E - modulus of elasticity of the cable, $\mathrm{H}_{\mathrm{s}}$ - tension force in the cable, F - cross-sectional area of the cable, 1 - the length of the cable, $\gamma_{1}$ - drag coefficient of the air, D cable diameter, $\eta$ - internal damping coefficient of the cable, $k_{n}$ - stiffness coefficient of the "n-th" damper, $c_{n}$ damping coefficient of the "n-th" damper, $\delta$ - Dirac delta function.

It was assumed the following load:

$$
p(x, t)=p_{0} e^{i \omega t}(9)
$$

The equation for "n-th" dynamic damper takes the following form:

$$
\begin{aligned}
\sum_{n=1}^{m} \frac{\partial^{2} w_{n}(t)}{\partial t^{2}} M_{n} & +\sum_{n=1}^{m} k_{n}\left(w_{n}(t)-w(x, t)\right) \delta\left(x-x_{n}\right) \\
& +\sum_{n=1}^{m} c_{n}\left(\frac{\partial w_{n}(t)}{\partial t}-\frac{\partial w(x, t)}{\partial t}\right) \delta(x \\
& \left.-x_{n}\right)=0
\end{aligned}
$$

where $M_{n}$ is the mass of "n-th" dynamic damper. After calculations, the solution of the system of equations (5), taking into consideration equation (1), the deflection function takes the form:

where:

$$
w(x, t)=\operatorname{Re}(w)+\operatorname{Im}(w)(11)
$$

$$
\begin{aligned}
& \operatorname{Re}(w) \\
& =p_{0} \sum_{j=1}^{k} \sum_{n=1}^{m} \sin \frac{j \pi x}{l} \frac{s_{1 n} \cos \omega t+s_{2 n} \sin \omega t}{s_{1 n}^{2}+s_{2 n}^{2}} \\
& \operatorname{Im}(w) p_{0} \sum_{j=1}^{k} \sum_{n=1}^{m} \sin \frac{j \pi x}{l} \frac{s_{1 n} \sin \omega t-s_{2 n} \cos \omega t}{s_{1 n}^{2}+s_{2 n}^{2}}
\end{aligned}
$$

In equations (12) and (13) $s_{1}$ and $s_{2}$ denote functions dependent on the known parameters of stiffness, damping, frequency domains, the mass of the cable and the masses of dampers.

\section{Numerical analysis}

Numerical analyses were performed for various parameters and the location of dampers. It was assumed the length of the steel cable $1=20 \mathrm{~m}$, cross section of the cable $\mathrm{F}=1,13 \mathrm{~cm}^{2}$, force of tension $\mathrm{H}_{\mathrm{st}}=20 \mathrm{kN}$.

Figure 2 shows that the use of the dynamic vibration damper reduces the dynamic displacement from the value of $62.12 \mathrm{~mm}$ to the value of $10,53 \mathrm{~mm}$. After using the damper placed in the middle of the cable, the displacement have been reduced by almost 6 times. The above reduction of vibration was achieved by choosing the optimal parameters as follows: $M_{1}=48,3 \mathrm{~kg}$, $k_{1}=13,03 \mathrm{kN} / \mathrm{m}, c_{1}=10,097 \mathrm{~N} * \mathrm{~s} / \mathrm{m}$.

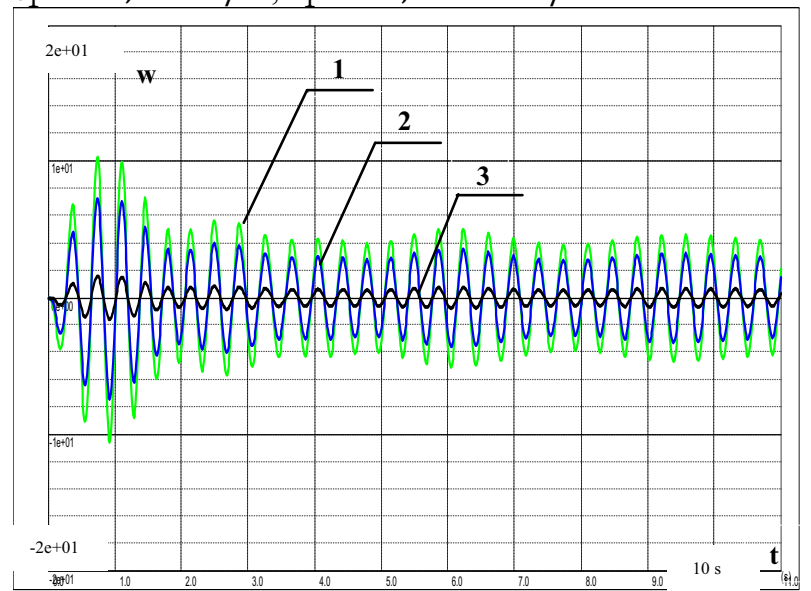

Fig.2. The dependence of displacement versus time for the vibration damper in the middle of the span. Curve 1 - vibration in the middle of the span of the cable, curve 2 - the vibration in the $1 / 4$ of span of the cable, curve 3 - vibrations close to the support.

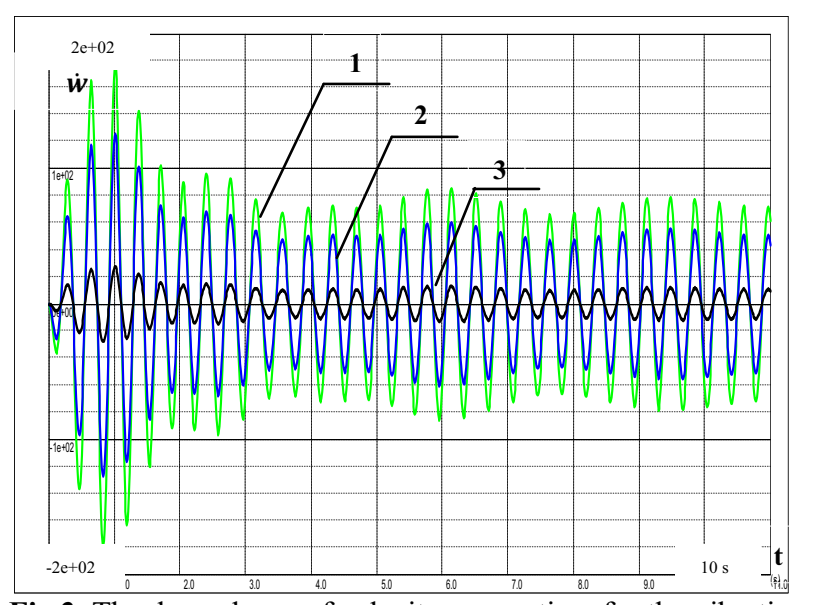

Fig.3. The dependence of velocity versus time for the vibration damper in the middle of the span. Curve 1 - vibration in the middle of the span of the cable, curve 2 - the vibration in the $1 / 4$ of span of the cable, curve 3 - vibrations close to the support.

Figure 3 shows the velocity versus time for the parameters of the damper as in the case shown in Figure 2. 
Figure 4 shows the acceleration versus time for the parameters of the damper as in the case shown in Figure 2.

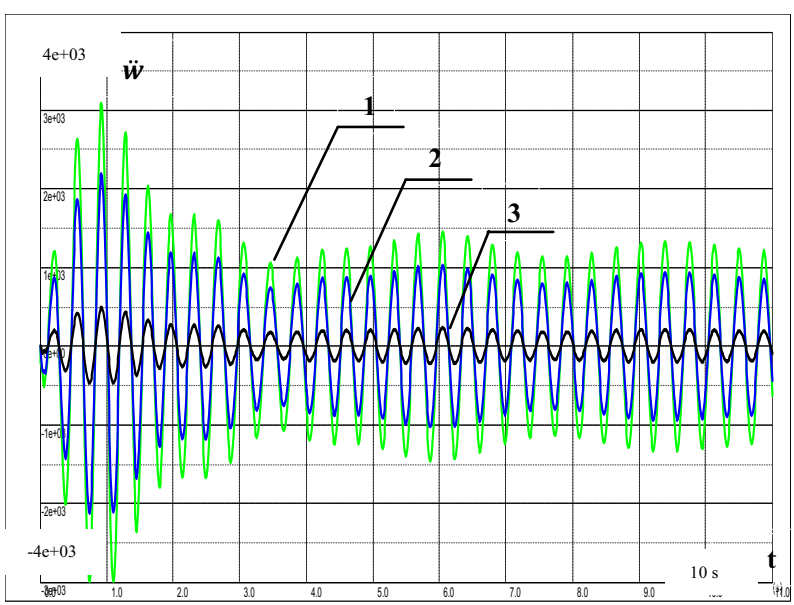

Fig.4. The dependence of acceleration versus time for the vibration damper in the middle of the span. Curve 1 - vibration in the middle of the span of the cable, curve 2 - the vibration in the $1 / 4$ of span of the cable, curve 3 - vibrations close to the support.

Figure 5 shows the displacement depending on the frequency of vibration of cable with damper and without damper.

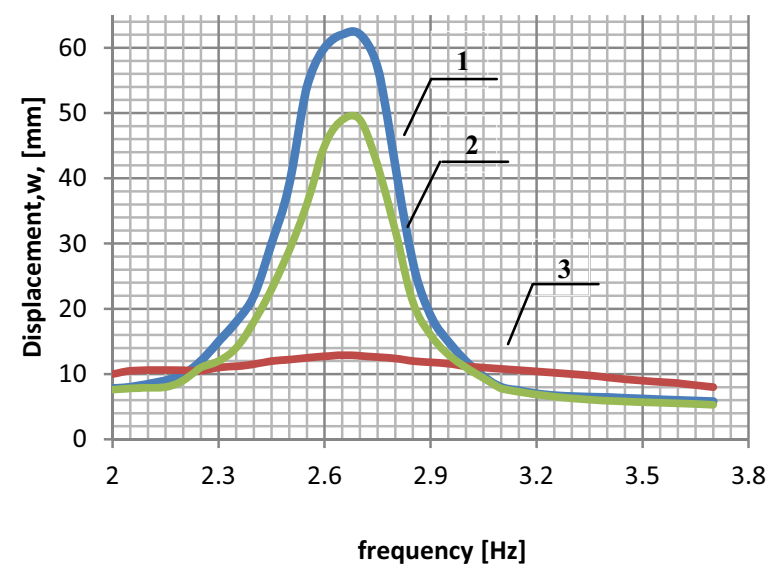

Fig. 5. The dependence of the displacement versus frequency, for a cable with damper and without damper. Curve 1 vibrations of the cable without damper, curve 2 - cable vibration for optimal parameters of the damper $M_{1}=48,3 \mathrm{~kg}$, $k_{1}=13,03 \mathrm{kN} / \mathrm{m}, \quad c_{1}=5,00 \mathrm{~N} * \mathrm{~s} / \mathrm{m}$, curve 3 - cable vibration for parameters of the damper $M_{1}=48,3 \mathrm{~kg}$, $k_{1}=13,03 \mathrm{kN} / \mathrm{m}, c_{1}=10,097 \mathrm{~N} * \mathrm{~s} / \mathrm{m}$.

Figure 6 shows the function of displacement versus the location of the vibration damper on the cable.

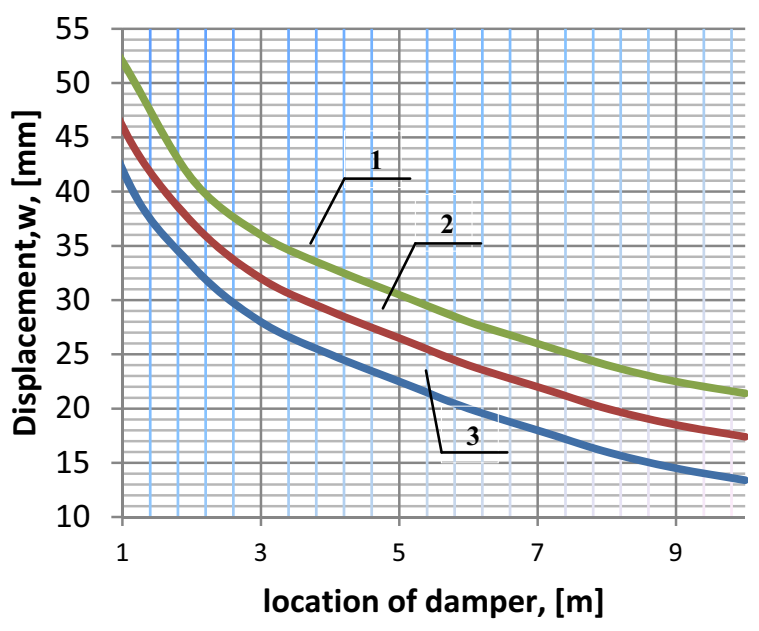

Fig.6. Dependence of displacement as a function of the location of the damper, Curve 1 - vibration of the cable for the parameters of the damper $M_{1}=48,3 \mathrm{~kg}, k_{1}=13,03 \mathrm{kN} / \mathrm{m}$, $c_{1}=2,50 \mathrm{~N} * \mathrm{~s} / \mathrm{m}$, curve 2 - vibration of the cable for the parameters of the damper $M_{1}=48,3 \mathrm{~kg}, k_{1}=13,03 \mathrm{kN} / \mathrm{m}$, $c_{1}=5,00 \mathrm{~N} * s / \mathrm{m}$, curve 3 - vibration of the cable for the parameters of the damper $M_{1}=48,3 \mathrm{~kg}, k_{1}=13,03 \mathrm{kN} / \mathrm{m}$, $c_{1}=10,097 \mathrm{~N} * \mathrm{~s} / \mathrm{m}$.

\section{Summary and Conclusion}

In the paper the importance of cable structures' analysis is emphasized. Cables in structures play an important supporting function. They are part of the construction of suspension bridges and the power transmission lines. From the point of view of the structural analysis, cables are important element in the design process to keep the sufficient ultimate and serviceability limit states. These constructions have large spans and low rigidity susceptible to deformation what was discussed in the paper. Because of low amplitudes often less than the diameter of the cable, these vibrations are slightly perceptible, but may lead to material fatigue and then to failure.

In the paper the influence of the parameters of the damper and the location on cable is shown and analysed. The proper location and optimization of damper's parameters is shown as requirement to reduce the vibration of the cable.

\section{References}

1. I.Gołębiowska, K.Peszyński, Reduction of Cable Vibrations Caused by Wind, Engineering Mechanics, 22th International Conference Engineering Mechanics, Svratka 2016

2. I.Gołębiowska, M.Dutkiewicz, B.Usewicz, Sposoby thmienia drgań napowietrznych linii elektroenergetycznych, TTS Technika Transportu Szynowego - 2015, 12, 2544-2548

3. B.M. Pacheco,Y. Fujino, A. Sulekh, Estimation Curve for Modal Damping in Stay Cables with 
Viscous Damper, J. Struct. Engrg., ASCE, 119(6), (1993).

4. K. Uno, S. Kitagawa, H. Tsutsumi, A.Inoue, S.Nakaya, A simple method of designing cable vibration dampers of cable-stayed bridges, J. of Struct.1 Eng., JSCE, Vol. 37A, pp.789-798, (1991)

5. H. Q. Wang, J. C. Miao, J. H. Luo, F. Huang, and L. G. Wang, The free vibration of long-span transmission line conductors with dampers, Journal of Sound and Vibr., 208(4):501 (1997).

6. H. Meynen, P.Verma, P. Hagedorn, M. Schafer, On the numerical simulation of vortexinduced vibrations of oscillating conductors, J.of Fluids and Struct., 21(1) (2005).

7. J.Hajduk, J. Osiecki, Ustroje ciegnowe, teoria $i$ obliczanie, WNT Warszawa (1970)

8. J.Vecchiarelli, I.G. Curie, D.G. Havard, Computational analysis of aeolian conductor vibration with a stockbridge-type damper, J. of fluids and struct. 14, 489-509 (2000) 